\title{
Multidimensional MRI-CT atlas of the naked mole-rat brain (Heterocephalus glaber)
}

\section{Fumiko Seki ${ }^{1,2 \dagger}$, Keigo Hikishima ${ }^{1,2+}$, Sanae Nambu ${ }^{3}$, Kazuo Okanoya ${ }^{4,5}$, Hirotaka J. Okano ${ }^{6}$, Erika Sasaki ${ }^{1,2}$, Kyoko Miura ${ }^{1,7}$ * and Hideyuki Okano ${ }^{1,8}$}

' Department of Physiology, Keio University School of Medicine, Tokyo, Japan

${ }^{2}$ Central Institute for Experimental Animals, Kanagawa, Japan

${ }^{3}$ Laboratory for Symbolic Cognitive Development, RIKEN Brain Science Institute, Saitama, Japan

${ }^{4}$ Japan Science and Technology Exploratory Research for Advanced Technology Okanoya Emotional Information Project, Saitama, Japan

${ }^{5}$ Department of Life Sciences, Graduate School of Arts and Sciences, The University of Tokyo, Tokyo, Japan

${ }^{6}$ Division of Regenerative Medicine, Jikei University School of Medicine, Tokyo, Japan

7 Precursory Research for Embryonic Science and Technology, Japan Science and Technology, Saitama, Japan

${ }^{8}$ Riken Keio University Joint Research Laboratory, RIKEN Brain Science Institute, Saitama, Japan

Edited by:

Angel Rodriguez, Universidad Politécnica de Madrid, Spain

\section{Reviewed by:}

Santiago Gonzalez, Universidad Politécnica de Madrid, Spain Consuelo Gonzalo-Martín, Universidad Politécnica de Madrid, Spain Jose-Maria Peña, Universidad Politécnica de Madrid, Spain

\section{*Correspondence:}

Kyoko Miura, Department of Physiology, Keio University School of Medicine, 35 Shinanomachi, Shinjuku, Tokyo 160-8582, Japan

e-mail:miura@z8.keio.jp

${ }^{\dagger}$ Fumiko Seki and Keigo Hikishima have contributed equally to this work.
Naked mole-rats have a variety of distinctive features such as the organization of a hierarchical society (known as eusociality), extraordinary longevity, and cancer resistance; thus, it would be worthwhile investigating these animals in detail. One important task is the preparation of a brain atlas database that provide comprehensive information containing multidimensional data with various image contrasts, which can be achievable using a magnetic resonance imaging (MRI). Advanced MRI techniques such as diffusion tensor imaging (DTI), which generates high contrast images of fiber structures, can characterize unique morphological properties in addition to conventional MRI. To obtain high spatial resolution images, MR histology, DTI, and X-ray computed tomography were performed on the fixed adult brain. Skull and brain structures were segmented as well as reconstructed in stereotaxic coordinates. Data were also acquired for the neonatal brain to allow developmental changes to be observed. Moreover, in vivo imaging of naked mole-rats was established as an evaluation tool of live animals. The data obtained comprised three-dimensional (3D) images with high tissue contrast as well as stereotaxic coordinates. Developmental differences in the visual system were highlighted in particular by DTI. Although it was difficult to delineate optic nerves in the mature adult brain, parts of them could be distinguished in the immature neonatal brain. From observation of cortical thickness, possibility of high somatosensory system development replaced to the visual system was indicated. 3D visualization of brain structures in the atlas as well as the establishment of in vivo imaging would promote neuroimaging researches towards detection of novel characteristics of eusocial naked mole-rats.

Keywords: cortical thickness, diffusion tensor imaging, eusociety, MR histology, postnatal development, rodents, sensory system, social behavior

\section{INTRODUCTION}

Naked mole-rats (Heterocephalus glaber), small fossorial rodents that live underground in the Horn of Africa (Somalia, Ethiopia, and Kenya), have been studied by researchers because they possess several unique biological characteristics. They are famous for their hyper-longevity: although the average body length of a naked mole-rat is only $8 \mathrm{~cm}$, their average lifespan is approximately 28 years (Buffenstein and Jarvis, 2002; Buffenstein, 2005, 2008; Edrey et al., 2011). They show considerable resistance to cancer (Buffenstein, 2005), are insensitive to pain caused by certain chemicals (capsaicin and acid; Park et al., 2008), and are tolerant

\footnotetext{
Abbreviations: 3D, three-dimensional; cc, corpus callosum; CT, computed tomography; DEC, direction-encoded color; DTI, diffusion tensor imaging; LGN, lateral geniculate nucleus; MRI, magnetic resonance imaging; RARE, rapid acquisition with relaxation enhancement; $\mathrm{SC}$, superior colliculus; $\mathrm{T}_{2} \mathrm{WI}, \mathrm{T}_{2}$-weighted image; VBM, voxel based morphometry.
}

to hypoxia (Larson and Park, 2009). Also, they are almost blind and are one of only two known eusocial mammals. They form underground colonies averaging 60-80 individuals. Their society consists of a single breeding female (queen), one to three breeding males (king(s)), and numerous non-reproductive subordinates (Jarvis, 1981). Subordinates do not show mating behaviors and act as "workers" or "soldiers" even when they have reached adulthood. The reproduction of subordinates is suppressed and they are instead engaged in foraging, maintaining the nest, caring for the young, and defending the colony from invasion by foreign enemies (Sherman et al., 1991).

To examine such unique and interesting features, detailed investigation of brain structures is of importance. The primary step is to establish the databases for anatomical characterization. The first atlas of naked mole-rats (Xiao et al., 2006) presents cytoarchitectural information with anatomical labeling, which have been 
widely used as the gold standard. However, the spatial relationships also need to be documented considering that brain structures are composed of three-dimensional (3D) organization. Magnetic resonance imaging (MRI) can resolve this issue because 3D images with high spatial resolution can be generated in a non-invasive fashion. MR histology techniques enable to generate 3D acquisition with isotropic voxels of several tens of micrometers (Petiet et al., 2008). The data are digitized, which allows them to be manipulated with ease, making it possible to perform the variety of image analyses unique to MRI atlases. Examples include an MRI mouse brain atlas combined with computed tomography (CT; Chan et al., 2007) and an MRI stereotaxic developmental brain atlas combined with diffusion tensor imaging (DTI) and CT (Aggarwal et al., 2009). An advantage of using non-invasive MRI and CT is to easily construct stereotaxic coordinates by combination of modalities without shrinkage of a specimen, which is difficult with histological methods. DTI, an advanced MRI technique, is capable of visualizing white matter fiber structures through analysis of the directional diffusion of water molecules within nerve tissue (Mori et al., 2001; Zhang et al., 2003). It enables researchers to evaluate neural fiber connectivity, which is a labor intensive exercise when performed using other techniques such as neuronal fiber tracers or tract-selective immunohistochemical staining (Fujiyoshi et al., 2007). Hence, MRI can provide a wealth of anatomical information through its capacity to depict the brain from multiple perspectives, such as in terms of neural morphometry and connectivity.

The aim of this study was to use these advanced MRI techniques to reveal the neuroanatomical structure of the naked mole-rat brain. Visualizing the shapes of brain structures and white matter bundles in 3D for the first time would allow us to highlight the diverse characteristics of this species, and might provide information about their unique sensory system and social system. The atlas would also contain the same kind of anatomical information from the neonatal brain MRI, making it possible to observe developmental changes. Furthermore, in vivo imaging was established, which was the critical step to elucidate a mechanism of brain development and a process of sexual maturation in future.

\section{MATERIALS AND METHODS DATA ACQUISITION OF THE ATLAS}

All procedures were performed in accordance with the Laboratory Animal Welfare Act and the Guide for the Care and Use of Laboratory Animals (National Institute of Health, Bethesda, MD, USA). All experiments were approved by the Animal Study Committee of Keio University in Japan (approval number; 09212-(1)). A male adult breeder ( 6 years old), a neonatal naked mole-rat (postnatal day 8 ), and a mouse (1 year old) for each were euthanized under deep anesthesia with isoflurane (Abbott Laboratories, Abbott Park, IL, USA) and chemically fixed with phosphate-buffered saline containing 4\% paraformaldehyde for 2 weeks. The skull was first imaged using a CT scan (R_mCT2, Rigaku Corp., Tokyo, Japan). With peak voltages of $90 \mathrm{kV}$, whole skull data were obtained within 2 min with isotropic resolution of $100 \mu \mathrm{m}^{3}$. This procedure was performed on the skull of the adult naked mole-rat. The specimens were then soaked in phosphate-buffered saline containing $0.5 \%$ sodium azide and the contrast agent gadopentetate dimeglumine (1 mM Magnevist; Schering, Berlin, Germany) for another 2 weeks to increase MRI tissue contrast. They were positioned in an acrylic tube filled with fluorinert (Sumitomo 3M Limited, Tokyo, Japan) to diminish the signal intensity due to the medium around the specimens. Brain MRI data were acquired using a 7T Biospec 70/16 scanner with CryoProbe (Bruker biospin MRI GmbH; Ettlingen, Germany). 3D ultra-high resolution $\mathrm{T}_{2}$-weighted images $\left(\mathrm{T}_{2} \mathrm{WI}\right)$ with isotropic resolution of $35 \mu \mathrm{m}^{3}$ were obtained by rapid acquisition with relaxation enhancement (RARE) with the following parameters: repetition time, $600 \mathrm{~ms}$; echo time, $16 \mathrm{~ms}$; number of averages, 1 ; and RARE factor, 2.

The next step was to acquire $3 \mathrm{D}$ ultra-high resolution DTI data with isotropic resolution of $67 \mu \mathrm{m}^{3}$ using a Stejskal-Tanner diffusion spin-echo sequence with the following parameters: repetition time, $600 \mathrm{~ms}$; echo time, $28 \mathrm{~ms}$; number of averages, 1 ; $b$-value, $2000 \mathrm{~s} / \mathrm{mm}^{2}$; number of motion probing gradient orientations, 12 axes. Table 1 shows our normalized diffusion gradient orientations. The total time taken to acquire data in each scan was approximately $20 \mathrm{~h}$.

\section{DATA ANALYSIS OF THE ATLAS}

Data on the skull and brain regions were semi-automatically extracted from the MRI and CT data using the "Segmentation Editor" in Amira software version 5.4 (Visage Imaging, Inc., San Diego, CA, USA). The skull was manually adjusted with affine transformation including translation and rotation by referring to stereotaxic coordinates as described in the stereotaxic mouse atlas (Franklin and Paxinos, 2007). Then, $\mathrm{T}_{2}$ WI and DTI were fused to the skull CT with stereotaxic coordinates by rigid body registration using "Affine registration" in Amira.

A DTI direction-encoded color (DEC) map was computed and fiber structures were tracked based on the FACT-like stream line algorithm (Mori et al., 1999) by the Diffusion Toolkit and TrackVis

\section{Table 1 | Normalized diffusion gradient orientations.}

\begin{tabular}{lrrr}
\hline Image volume & \multicolumn{3}{c}{ Gradients } \\
\cline { 2 - 4 } & \multicolumn{1}{c}{$\boldsymbol{x}$} & $\boldsymbol{y}$ & \multicolumn{1}{c}{$\boldsymbol{z}$} \\
\hline 1 & 0.0000 & 0.0000 & 0.0000 \\
2 & 0.8944 & 0.0000 & 0.8944 \\
3 & 0.0000 & 0.4472 & 0.8944 \\
4 & 0.4472 & 0.8944 & 0.0000 \\
5 & 0.8944 & 0.4472 & 0.0000 \\
6 & 0.0000 & 0.8944 & 0.4472 \\
7 & 0.4472 & 0.0000 & 0.8944 \\
8 & 0.8944 & 0.0000 & -0.4472 \\
9 & 0.0000 & -0.4472 & 0.8944 \\
10 & -0.4472 & -0.8944 & 0.0000 \\
11 & 0.8944 & -0.4472 & 0.0000 \\
12 & 0.0000 & 0.8944 & -0.4472 \\
13 & -0.4472 & 0.0000 & 0.8944
\end{tabular}


software (Martinos Center for Biomedical Imaging, Massachusetts General Hospital $)^{1}$ with the following parameters: fiber turning angle for ending tracking, $35^{\circ}$; rendering with line mode. The diffusion tensor can be represented as an ellipsoid, where a proton at the center of the voxel has an equal probability of diffusing to any point in that ellipsoid. For delineation of specific bundles by tractography, selective bundles were segmented by manually placing regions of interest with reference to the newly generated DEC map and the mouse brain atlas (Franklin and Paxinos, 2007) with multiROI approach (Conturo et al., 1999; Huang et al., 2004). Major brain regions (caudate putamen, hippocampus, and cerebellum) were semi-automatically segmented using the "Segmentation Editor" and reconstructed using the 3D rendering module based on polygon-based rendering in Amira. For reference, volumes of all segmented regions in both the adult and neonatal brain, as well as the whole brain volumes of brain in vivo were calculated the sum of tetrahedral joining surface triangles to the origin using "surface area” measurements in Amira (Brandt et al., 2005; Ullmann et al., 2010; Hikishima et al., 2011).

Three-dimensional visualization made it possible to conduct image analysis focusing on cortical distribution. To identify any features specific to naked mole-rats, the MRI of the adult mouse brain was used for comparison. The cerebral cortex of naked mole-rats (adult and neonatal) as well as those of the mouse were semi-automatically segmented with reference to atlases of naked mole-rats (Xiao etal., 2006) and mice (Franklin and Paxinos, 2007), respectively. Cortical thickness was computed from the 3D cortical area. Mapping of cortical thickness was performed by computing the distance from the vertex to the point where the normal intersected the closest triangle in each vertex on the segmented cortical surface (Zhang etal., 2003; Huang etal., 2009). Knowledge of localized cortical areas was available for the mouse brain; information about the cortical map specific to the sensory system was thus added to the resulting $3 \mathrm{D}$ image by manual drawing, with references to the 3D atlases (Hjornevik et al., 2007; Lein et al., 2007; Hawrylycz et al., 2011), and the mouse brain atlas (Franklin and Paxinos, 2007).

\section{DATA ACOUISITION OF IN VIVO IMAGING}

In vivo imaging was performed on 4 naked mole-rats in a colony; 5-years-old female breeder; 41 g, 5-years-old male breeder; $40 \mathrm{~g}$, 1-year old two male non-breeders 28,38 g, respectively. Naked mole-rats were anesthetized with a mixture of $2.0 \%$ of isoflurane and oxygen at a constant rate (Herold et al., 1998) using a ventilator, SN-480-7 (Shinano, Tokyo, Japan). Both rectal and skin temperatures were monitored considering that naked mole-rats were heterothermic animal. Their temperatures were maintained around 29-32 centigrade, which was the appropriate condition for resting states of naked mole-rats (Jarvis, 1978). When their temperatures were declined, a hot-air type heater was put on for several minutes. Moisturizing gel was covered on the dorsum to prevent from drying.

An imaging coil with inner diameter of $38 \mathrm{~mm}$ was fitted to their head size was thus selected for in vivo MRI. The same sorts

\footnotetext{
${ }^{1}$ http://www.trackvis.org
}

of image contrast with the atlas were acquired: $\mathrm{T}_{2} \mathrm{WI}$ with coronal section by RARE with the following parameters: repetition time, $3000 \mathrm{~ms}$; echo time, $34 \mathrm{~ms}$; number of averages, 25; and RARE factor, 8 and respiratory gating, and DTI with coronal section by eight-shots echo planner imaging sequence based on the Stejkkal-Tanner diffusion preparation with following parameters: repetition time, $3000 \mathrm{~ms}$; echo time, $41 \mathrm{~ms}$; number of averages, 4; $b$-value, $1000 \mathrm{~s} / \mathrm{mm}^{2}$; number of motion probing gradient orientations, 12 axes, and respiratory gating. The resolution of acquired images was as follows: $\mathrm{T}_{2} \mathrm{WI}$ : in plane resolution, $125 \mu \mathrm{m}^{2}$; slice thickness, $500 \mu \mathrm{m}$; DTI: in plane resolution, $125 \mu \mathrm{m}^{2}$; slice thickness, $750 \mu \mathrm{m}$. The total time required for an experiment was $1.5 \mathrm{~h}$. Acquired data were computed in the same fashion as the atlas.

\section{RESULTS}

Images of the skull of the adult naked mole-rat were first acquired with a CT scan. Figure 1 shows a volume-rendered skull image with stereotaxic coordinates for the landmarks Bregma and lambda, and the interaural line, along with 3D brain images to reveal the approximate locations of major structures. It demonstrates that the skull was adjusted according to stereotaxic coordinates; the side view confirmed that the Bregma was parallel to the lambda.

Figure 2 shows slices in sagittal, horizontal, and coronal planes from high resolution $\mathrm{T}_{2}$ WIs (i.e., MR histology) and the DTI DEC maps with labeling of major structures. High soft-tissue contrast made various anatomical structures visible in the images. Both sets of data were combined with the skull CT data, so that the brain data included stereotaxic coordinates, as demonstrated by the MR histology. In the DTI data, visualization with different colors made it possible to localize white matter bundles, such as the medial and lateral parts of the habenular commissure, which could barely be discriminated in the MR histology coronal slices. The alphabetical listing of all abbreviations with corresponding structures is available in Table 2. The study focused on delineating the visual system by DTI, taking into consideration the fact that naked mole-rats are almost blind. In the brain of the adult naked mole-rat, the lateral geniculate nucleus (LGNs) and the optic tract could be identified from the coronal plane of the 2D DTI DEC map. The data presented are stored in Nifti compressed format ${ }^{2}$ and can be downloaded from the CT-MRI naked mole-rat brain database ${ }^{3}$.

Also, Figure 2 shows slices in coronal plane from in vivo $\mathrm{T}_{2} \mathrm{WIs}$ and the DTI DEC maps of the male breeder with labeling of major structures. Images demonstrated that the major regions could be sufficiently identified from in vivo $\mathrm{T}_{2}$ WIs and DTI DEC maps. Piriform cortex could be particularly visible in the coronal plane of the $\mathrm{T}_{2} \mathrm{WIs}$. In the DTI DEC maps, cingulum and external capsule could be identified. The volumes of whole brain from four individual images are shown in Table 3.

Figure 3 shows 3D images of major white matter tracts obtained with DTI tractography (side, top, and bottom views); the structures have been colored to make them easy to identify. This

\footnotetext{
${ }^{2}$ http://nifti.nimh.nih.gov/

${ }^{3}$ http://131.113.177.229/mri/
} 


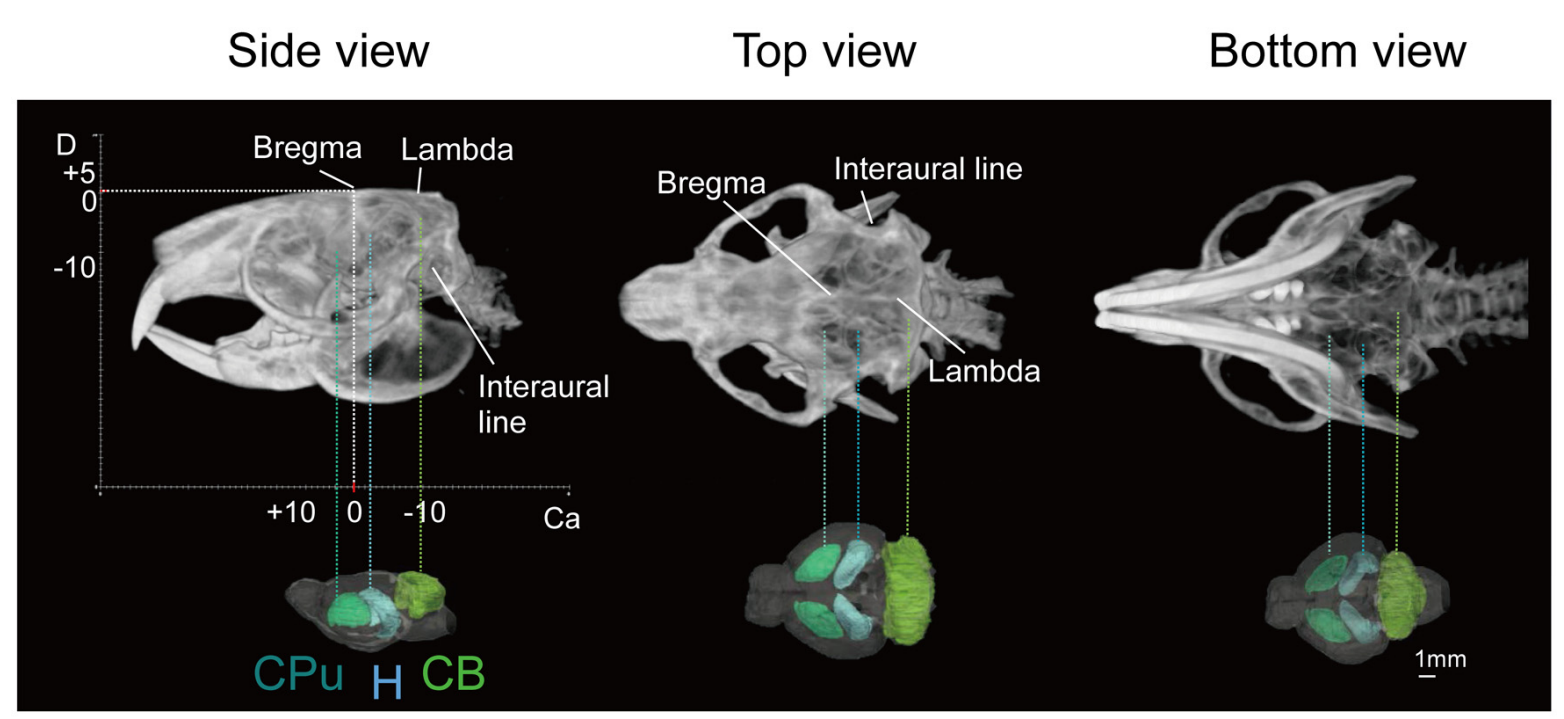

FIGURE 1 | Volume-rendered skull images with stereotaxic coordinates (left, side view; center, top view; right, bottom view) together with whole brain images displaying the approximate locations of major regions caudate putamen (CPu, light green), cerebellum (CB, green), and hippocampus (H, light blue). Scale bar $=1 \mathrm{~mm}$.

approach made it possible to capture the spatial relationships between white matter tracts to detect possible neural connections between regions. 3D delineation of optic nerve was difficult while the optic tracts could be drawn with DTI tractography.

The series of data obtained with the adult brain were also acquired for the neonatal brain, as shown in Figure 4. Neonatal brain images in the form of both MR histology and a DTI DEC map were shown in the same layout as for the adult; representative one slice each from sagittal, horizontal, and coronal planes with labeling for comparison. The contrast of MR histology was poor due to the lack of myelination compared with the adult brain. Although there were far fewer identifiable structures in the neonatal brain than the adult brain, the commissural tracts could be seen in the sagittal plane of the DTI DEC map.

Images from DTI tractography of the neonatal brain are shown in Figure $\mathbf{5}$ as side, top, and bottom views. The same color scheme was applied to the neonatal brain as was used for the adult brain. While the shape of the neonatal brain as a whole shows that it is in the process of growing, the shapes of the hippocampus and the caudate putamen seemed similar to those of the adult, respectively. The volumes of segmented regions were easily measurable due to the acquisition of $3 \mathrm{D}$ digital data. Table 4 shows the volumes of five major regions of both adult and neonatal brains, and suggests that the degree of development might differ depending on the region. This could also apply to delineated white matter structures: white matter tracts of the cerebral peduncle were delineated but those of the middle cerebellar peduncle were not. The development of the visual system can also be compared on the basis of its degree of delineation. Interestingly, as in the adult brain, the optic tract and LGN could be identified in the DTI DEC map of the neonate; however, it was only in the neonatal brain that the optic nerve $(2 n)$, which was colored light blue, could be delineated before crossing at the chiasm.
Figure 6 shows 3D images of cerebral cortical thickness in the mouse brain overlaid with a cursory drawing that maps the major sensory systems, which allows comparison with images of the adult and neonatal naked mole-rat brain that were analyzed in the same fashion. The degree of cortical thickness is represented by color. Dark blue corresponds to $0.2 \mathrm{~mm}$ (thinnest), while red corresponds to $1.8 \mathrm{~mm}$ (thickest). The distribution of cortex was similar in the adult naked mole-rat and the mouse in that the rostral areas were thick; however, the caudal areas, which the visual cortex occupies, were thicker in the naked mole-rat than in the mouse. In addition, images of the corpus callosum (cc) were added to the images of the adult and neonatal naked mole-rat brain to determine whether cortical development corresponds to the development of the cc. In the adult brain, the fiber structures of the cc innervated both sides of the cortex. By contrast, in the neonatal brain, the connection was immature and projections to rostral as well as caudal areas were observed.

\section{DISCUSSION}

We constructed a 3D MRI-CT brain atlas and in vivo imaging for neuroimaging research of the naked mole-rat from multiple perspectives. Three-dimensional MR histology were used to visualize anatomical features by means of high soft-tissue contrast, representing an addition to the histological atlas (Xiao et al., 2006). The DTI DEC maps emphasized fiber bundles, and the spatial relationship between the white matter tracts and major brain regions was demonstrated by DTI tractography. Also, our datasets were easily incorporated into $3 \mathrm{D}$ reconstructions, which meant that we could observe differences in the distribution of cortical thickness between the naked mole rat and the mouse. Combining skull CT images with brain images means that our atlas contains stereotaxic coordinates with contrast between bone and brain tissues. This visualization will be valuable when precise targeting procedures of 


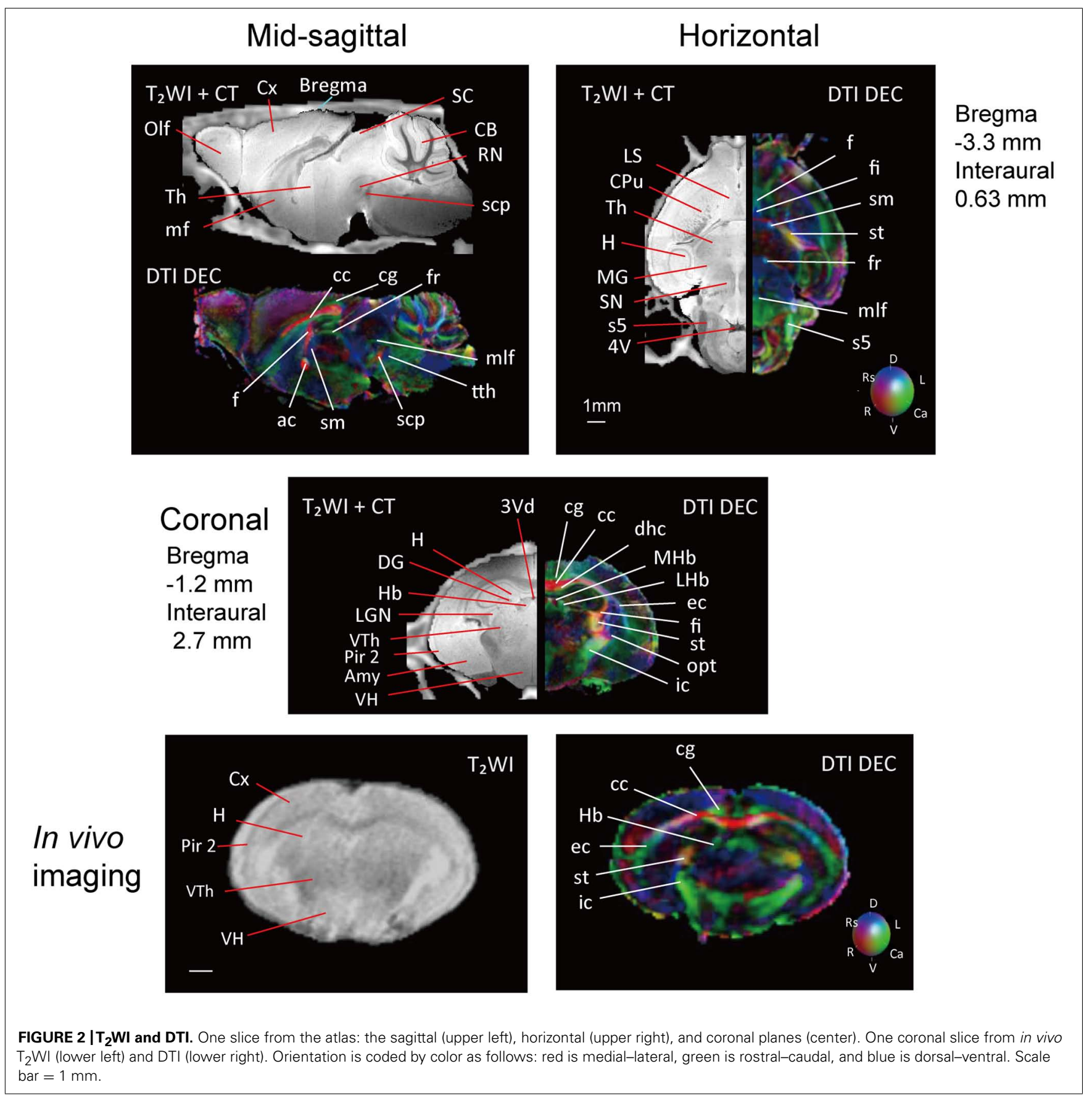

the brain (e.g., electrode implantation and injection of chemical substances) are required (Aggarwal et al., 2009). The anatomical information acquired will also provide information about the morphology of the sensory and social system, which could possibly be developed further.

\section{THE DEVELOPMENT OF THE MAJOR WHITE MATTER TRACTS AND THE VISUAL SYSTEM}

It is possible to compare the development of segmented white matter tracts by referring to the mouse MRI atlas (Chuang et al., 2011). By 8 days after birth, the volume of the major white matter tracts had reached $50 \%$ of their estimated volume in the adult. The tracts include the hippocampal commissure, anterior commissure, $\mathrm{cc}$, fornix, stria medularis, and fasciculus retroflexus, all of which could be delineated by tractography (Chuang et al., 2011). Given that the neonatal brain of the naked mole-rat was examined 8 days after birth, at a similar time point to that of the mouse, there may not be any obvious differences in segmented fiber structures; all white matter tracts except the fornix could also be delineated in the naked mole-rat.

On the other hand, the optic nerve in the mouse could be clearly visualized from embryonic day 12 (Chuang et al., 2011), 
Table 2 | Alphabetical lists of all abbreviations with corresponding structures; left, $\mathbf{T}_{2}$ WI; right; DTI.

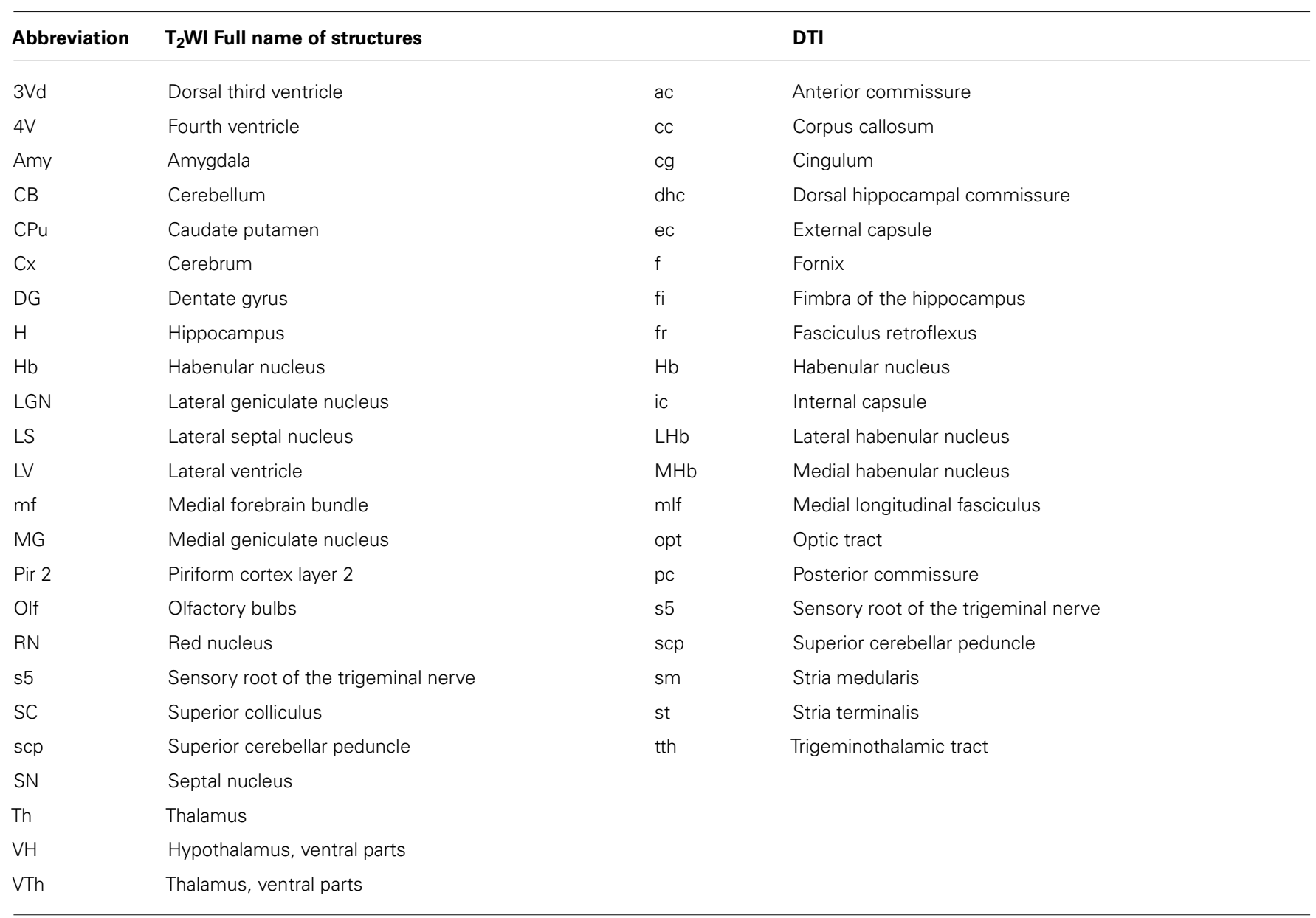

Table 3 | Volumes of the whole brain in male adult and neonatal postmortem specimens, and four subjects; a female adult breeder (queen), a male adult breeder (king), two male non-breeders ( $\sigma^{\top}$ worker).

\begin{tabular}{|c|c|c|c|c|c|c|}
\hline & Adult & Neonate & Queen & King & o'Worker & orWorker \\
\hline Volume $\left(\mathrm{mm}^{3}\right)$ & 484.9 & 249.0 & 484.1 & 493.1 & 489.6 & 484.1 \\
\hline
\end{tabular}

whereas it was unclear either in neonatal or adult stages of naked mole-rats. A previous study has confirmed that the visual system of the naked mole rat has regressed due to their underground life (Hetling et al., 2005), yet it is uncertain from previous findings to what degree the naked mole-rat may retain a visual system. Despite lacking the ability to recognize shapes, which requires higher-order function, naked mole-rats have retained the muscular function of the iris compared with other genera of mole rats, suggesting that they have some ability to detect light (Nikitina et al., 2004). The presence of the olivary pretectal nucleus (Crish et al., 2006a) as well as differences in locomotor activity (due to circadian rhythms) during exposure to periodic light/dark changes (Riccio and Goldman, 2000a,b) has also been confirmed in naked mole-rats.

The optic tracts could be delineated; however, the optic nerve could not be delineated by DTI, indicating that the visual system of the adult naked mole-rat has undergone regression. On the other hand, parts of the optic nerve and the optic tract could be visualized in the neonatal brain. This implies that the development of the optic nerve may have some unique features; indeed, postnatal development of the eye is considered to be irregular, especially in the case of the lens given loss of crystallins in lens of adults which was present in neonatal were suggested (Nikitina et al., 2004).

The possibility that naked mole rats retain a visual system to some degree is also of interest, in particular whether the development of the visual system may show plasticity in response to exposure to different amounts of light after birth (Daw, 2006). Naked mole-rats in our laboratory were raised in an environment in which a certain amount of light is always present. Modifications such as an increase in brightness or limiting light exposure to $12 \mathrm{~h}$ per day may affect the development of the visual system. If parts 


\section{Side view}

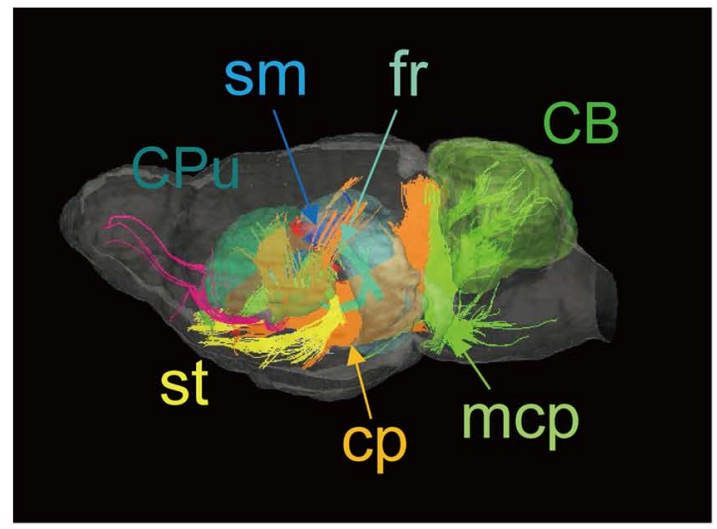

\section{Top view}

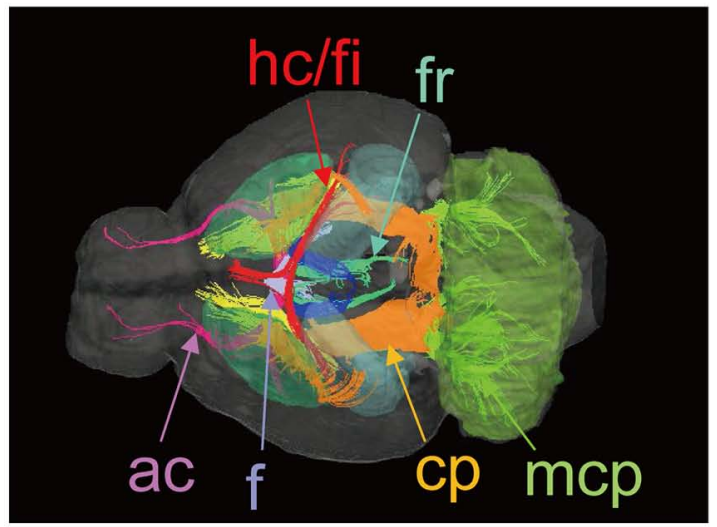

FIGURE 3 | Tractography showing the white matter tracts in 3D (upper, side view; lower left, top view; lower right, bottom view). ac, anterior commissure (pink); cp, cerebral peduncle (orange); f, fornix (light purple); fr, fasciculus retroflexus (light green); hc/fi, hippocampal commissure/fimbria
Bottom view

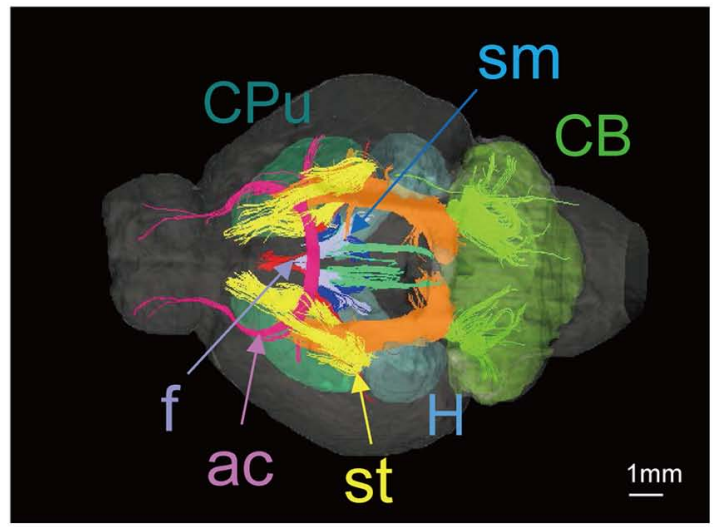

(red); mcp, middle cerebellar peduncle (green); sm, stria medularis (blue); st, stria terminalis (yellow) together with the caudate putamen (CPu; light green) cerebellum (CB; green), and hippocampus $(\mathrm{H}$; light blue). Scale bar $=1 \mathrm{~mm}$. of the visual system function, light stimuli may affect the development of not only the visual system but also brain structures associated with visual processing such as the visual cortex, LGN, and SC.

Magnetic resonance imaging would make it possible to follow the development of the visual system because it would permit continuous observation of the same subject. In fact, atlases of the developing brain in both rodents (Petiet et al., 2008; Aggarwal et al., 2009; Chuang et al., 2011) and non-human primates (Hikishima et al., 2013) have been available. Evaluation of developmental process in naked mole-rats may be important for understanding brain development and the visual system, and for investigating how the optic nerve develops.

In connection with this, the visual system can also be examined with respect to cortical distribution. The primary motor cortex (M1) is the thickest, while the primary visual cortex (V1) is the thinnest in humans, non-human primates, and rodents (Brodmann, 2006). M1 in both mouse and the naked mole-rat are the same distribution, but the cortex in the caudal area occupied by visual cortex seemed to be thicker in the naked mole-rat than in the mouse, according to a number of mouse anatomic atlases (Franklin and Paxinos, 2007; Hjornevik et al., 2007; Lein et al., 2007; Hawrylycz et al., 2011). This contradiction may be linked with data indicating that the somatosensory cortex has replaced significant parts of the visual cortex (Catania and Remple, 2002). Precise localization of cortical thickness would enable researchers to clarify the extent to which cortical distribution is unique such as in visual regions. It may also be possible to correlate the development of the cerebral cortex and the fiber bundles extending to the cortex, given that the cc and visual cortex reportedly show plasticity in response to visual stimuli (Pietrasanta et al., 2012).

For further investigation towards revealing morphological properties discussed in this study, it is essential to obtain more precise anatomical information of the naked mole-rat brain, considering that the data of the present atlas were limited to a single specimen. One reason for this, which we are currently trying to address, is the difficulty of securing greater numbers of naked mole-rats due to their slow reproduction. This atlas does not reflect 


\section{Mid-sagittal}

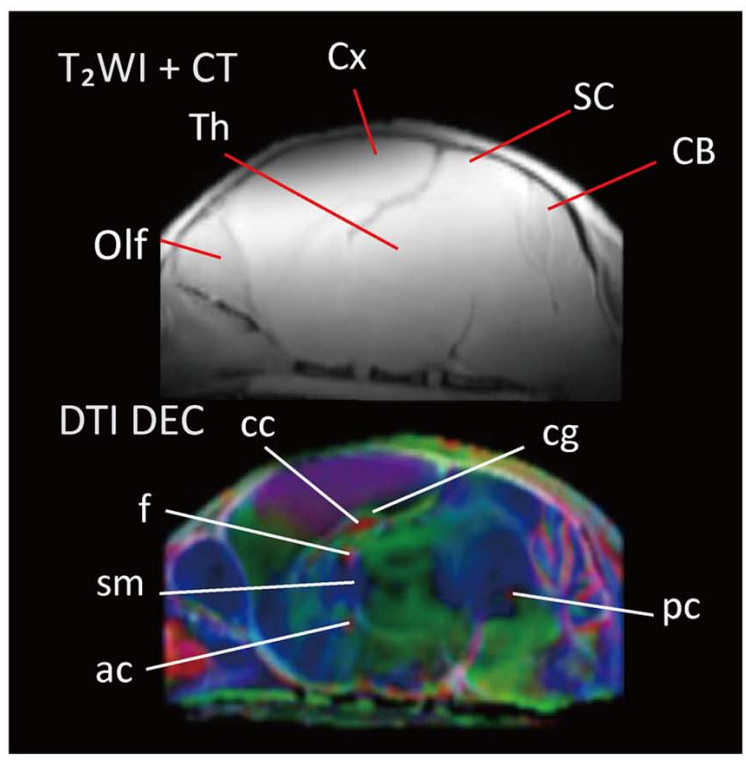

\section{Horizontal}

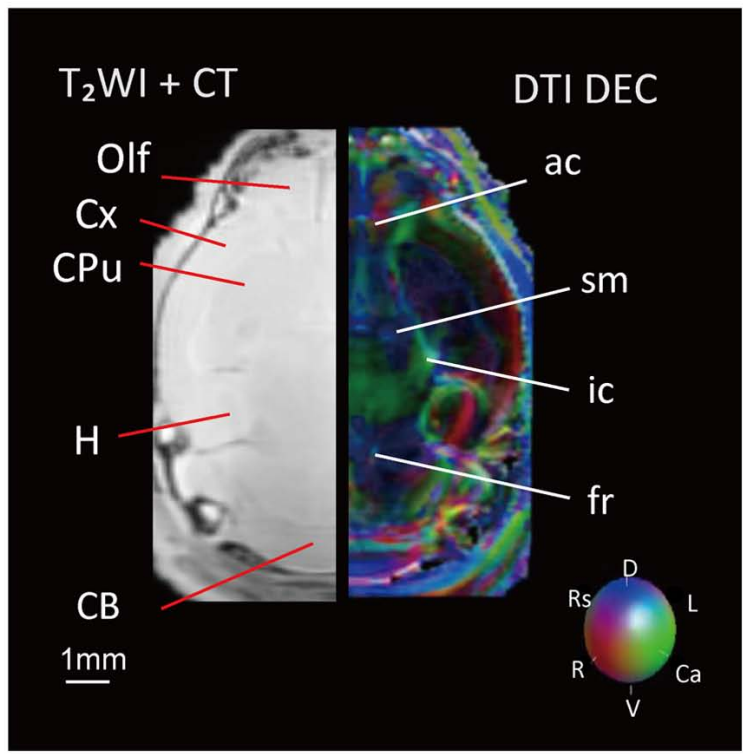

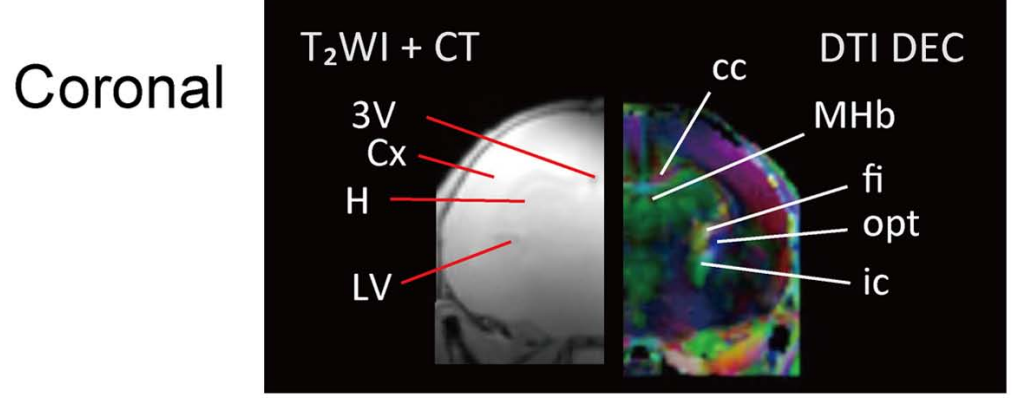

FIGURE $\left.4\right|_{2} \mathrm{~T}_{2} \mathrm{WI}$ and DTI of the neonatal brain in three different planes; the sagittal (upper left), horizontal (upper right), and coronal planes (center). Orientation is coded using the same color scheme as in the adult brain: red is medial-lateral, green is rostral-caudal, and blue is dorsal-ventral. Scale bar $=1 \mathrm{~mm}$.

\section{Side view}

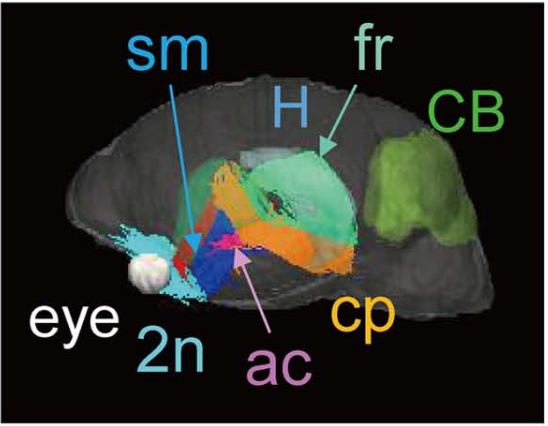

\section{Top view}

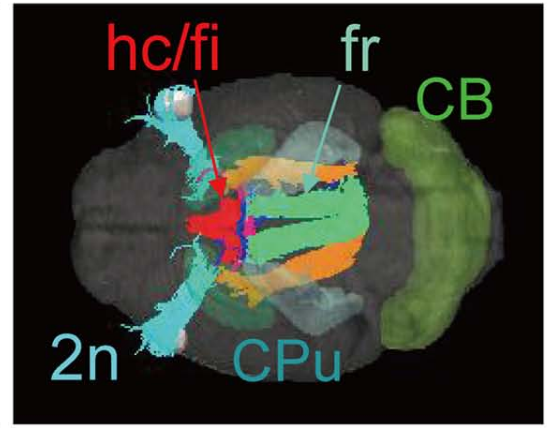

\section{Bottom view}

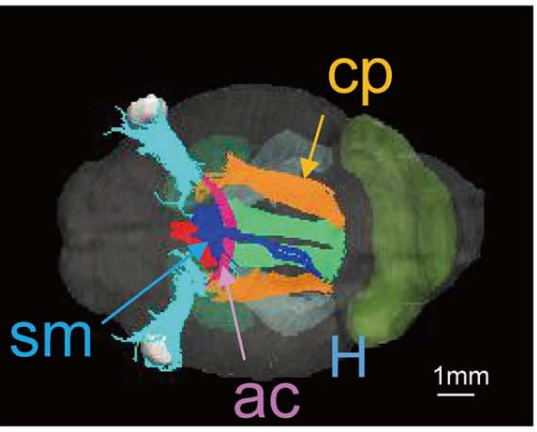

FIGURE 5 | Tractography of the neonatal brain (left, side view; center, top view; right, bottom view). $2 n$, optic nerve (light blue); ac, anterior commissure (pink); $c p$, cerebral peduncle (orange); fr, fasciculus retroflexus (light green); hc/fi, hippocamal commissure/fimbria (red); sm, stria medularis (blue), together with the caudate putamen ( $\mathrm{CPu}$, light green), the cerebellum (CB, green), the hippocampus ( $\mathrm{H}$, light blue), and the eye (white). Scale bar $=1 \mathrm{~mm}$. 
Table 4 | Volumes of the whole brain, cerebral cortex, cerebellum, caudate putamen, and hippocampus in the adult and neonatal naked mole-rat.

\begin{tabular}{lll}
\hline Structure & Adult $\left(\mathbf{m m}^{\mathbf{3}}\right)$ & Neonate \\
\hline Whole brain & $\mathbf{3})$ \\
Cerebral cortex & 484.9 & 249.0 \\
Cerebellum & 110.4 & 74.1 \\
Caudate putamen & 57.9 & 16.6 \\
Hippocampus & 16.9 & 7.3 \\
\hline
\end{tabular}

individual differences such as sex differences or indeed the presence/absence of breeding ability, although there is reportedly no significant variation in brain weight (Xiao, 2007) or body weight (Pinto et al., 2010) between male breeders and non-breeders. The development of a population-averaged atlas using in vivo imaging, as has been done for other model animals (Black et al., 2001; Watanabe et al., 2001; Hikishima et al., 2011) is the crucial task.

\section{REGIONS RELEVANT TO SOMATOSENSORY PROCESSING}

This study demonstrated the utility of DTI for investigating neural connectivity. For the brain of the naked mole-rat, this strength can be utilized to examine the networks of the midbrain, especially the superior colliculus (SC), because the unique functions of the naked mole-rat SC have been well studied. We focused on the SC because it has an important role in processing visual information, especially eye and/or head and body movements. The superficial layers are responsible for receiving and processing visual inputs (May, 2006) and are severely atrophied in naked mole-rats (Crish etal., 2006b) and blind mole rats (Cooper et al., 1993). Furthermore, the SC receives somatosensory information from tactile hairs and projects to the facial nucleus (Izraeli and Porter, 1995; Miyashita and Mori, 1995; Hattox et al., 2002). Processing of somatosensory, auditory, and inhibitory inputs in the intermediate and deeper layers is integrated with visual information to acquire spatial information and elicit a concerted action (Drager and Hubel, 1975). Given that the reduction of SC volume in naked mole-rats is considered to be the result of atrophy of the superficial layers (Crish et al., 2004), it seems that their SC is specialized for somatosensory oriented behaviors, in contrast to other sighted rodents in which the SC functions in visual processing (Crish et al., 2006b). This implies that the anatomical structure of the SC in naked mole-rats is unique.

Naked mole-rats have a highly developed somatosensory system to detect external stimuli. In mammals, a major source of somatosensory information comes from the facial vibrissae. Naked mole-rats also have these vibrissae on their face, but in addition they have approximately another 40 tactile hairs on each side of their body, which have similar functions to the facial vibrissae (Crish et al., 2003). Information from the tactile hairs can guide accurate and appropriate behaviors (Crish et al., 2003), and the SC is involved in vibrissae orienting behaviors (Crish et al., 2006b). Since there is a tight connectivity of neural circuits between the afferent nerve from body and the SC, the connectivity of the SC

\section{Cortical thickness}

\section{Corpus callosum}
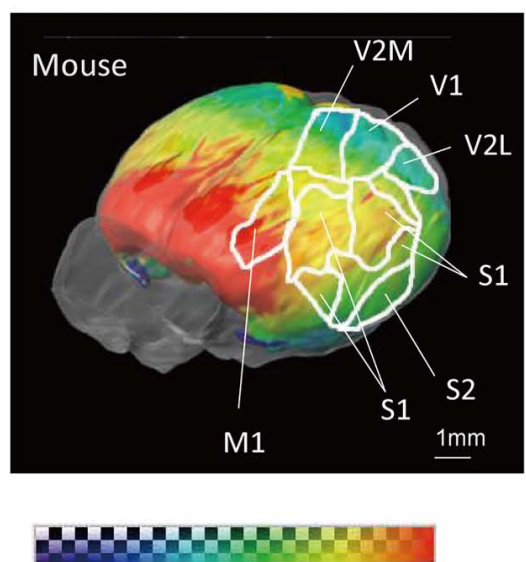

$0.2 \mathrm{~mm}$
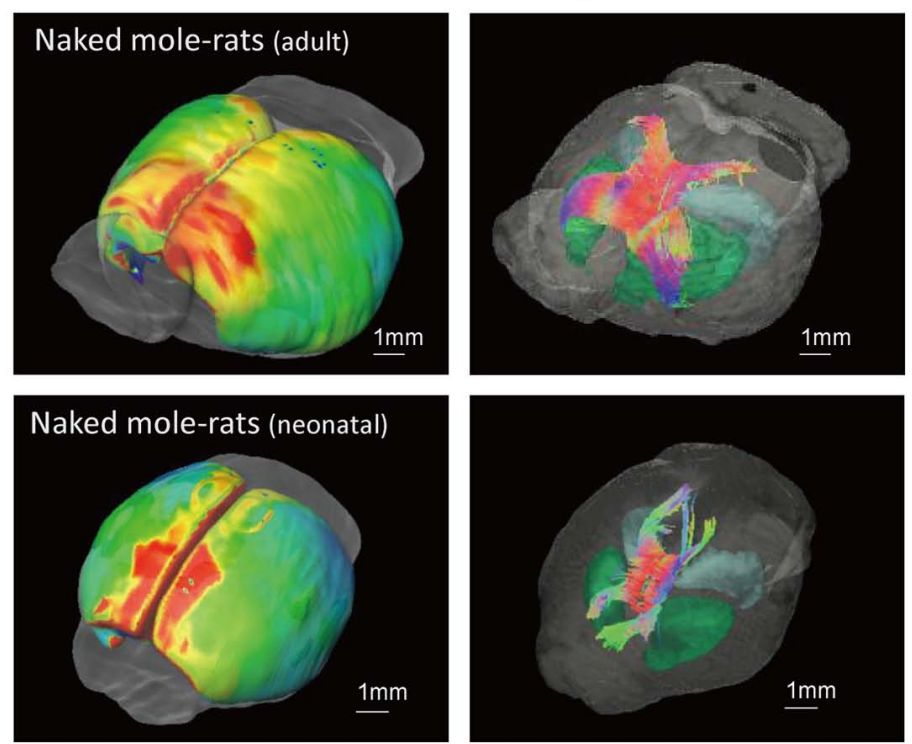

FIGURE 6 | 3D surface maps showing cortical thickness and images of corpus callosum of the adult (above right) and neonatal (below right) naked mole-rats brain, and the mouse brain that maps major sensory systems (left). Thickness is indicated by color as follows: (dark blue, $0.2 \mathrm{~mm}$ to red, $1.8 \mathrm{~mm}$ ). Whole brain images with the corpus callosum are shown to the right. Colors reveal the directions of the tracts (red, right-left; green, cranial-caudal; blue, ventral-dorsal). M1, primary motor cortex; S1, primary somatosensory cortex; S2, secondary somatosensory cortex; $\mathrm{V} 1$, primary visual cortex; V2M, secondary visual cortex medial area; V2L, secondary visual cortex lateral area. Scale bar $=1 \mathrm{~mm}$. 
may show unique features. For example, it is of interest whether the connection between superficial layers and the optic nerve can be visualized. In future studies, it is worth probing the neural network around the midbrain in detail by applying advanced diffusion tractography such as diffusion spectrum imaging (Wedeen et al., 2012) combined with neural tracing.

As somatosensory occupation was suggested from observation of cortical thickness, the dominance of the somatosensory system has also been observed from previous studies (Crish et al., 2003; Park et al., 2003), with inputs derived not only from unique tactile hairs, but also from the teeth (Catania and Remple, 2002). In addition to general uses of the teeth such as in digging, the huge teeth of the naked mole-rat have another role in aggressive facial behaviors (Sherman et al., 1991), which may be associated with hierarchy formation (Brecht and Freiwald, 2012). Queens use aggressive facial behaviors to govern their society (Reeve, 1992; Clarke and Faulkes, 1997), and members of this society may in turn show morphological differences depending on their social status. Given that neural changes might occur during the shift from non-breeder to breeder (Holmes et al., 2007), it is important to examine morphological changes in structures throughout the brain during a promotion of social status (i.e., from non-breeder to breeder).

In human subjects, analyses of subtle differences in brain anatomy such as sex difference (Good et al., 2001) or life-span brain development (Giorgio et al., 2010a,b; Groeschel et al., 2010) have been examined using VBM. In small animals subjects, as was conducted with naked mole-rats in this study, the establishment of in vivo MRI combined with the atlas in stereotaxic coordinates will enable to apply VBM with a statistical atlas in future. Following development of VBM for naked mole-rats allows us to evaluate longitudinal changes by focusing the process of sexual maturation, which is a prominent characteristic of the exceptional eusocial mammal.

\section{CONCLUSION}

Naked mole-rats have a number of as yet poorly characterized interesting features, and this atlas as well as the in vivo imaging techniques will play an important role in their analysis by using anatomical information from various different perspectives. We detected atypical development of the visual system via a DTI and differences in cortical distribution between the naked mole-rat and the mouse, which might result from altered development of the somatosensory system relative to the visual system. Further examination of the sensory system by MRI would be valuable as it could be used to non-invasively track visual system development as well as to precisely localize cortical distribution in specific subjects, and could be coupled with the construction of VBM based on the present atlas and in vivo imaging, for the analysis of morphological differences of social status in a hierarchical classification.

\section{ACKNOWLEDGMENTS}

The authors would like to give special thanks to Dr. Koichi Matsuo and Dr. Yasunari Takada for significant support of CT data acquisition, and Dr. Tsuyoshi Hachiya for technical support to upload MRI data to the web server. This work was supported by grants from the JSPS research fellowships for Young Scientists (SPD) and Kanae foundation for the promotion of medical science to Kyoko Miura, and from "Funding Program for Worldleading Innovative R\&D on Science and Technology" to Hideyuki Okano.

\section{AUTHOR CONTRIBUTIONS}

Fumiko Seki and Keigo Hikishima conducted research; Sanae Nambu, Kazuo Okanoya, Hirotaka J. Okano, Erika Sasaki, and Hideyuki Okano provided essential materials. Fumiko Seki, Keigo Hikishima, and Kyoko Miura wrote the paper; Kyoko Miura and Hideyuki Okano had primary responsibility for final content. All authors read and approved the final manuscript.

\section{REFERENCES}

Aggarwal, M., Zhang, J., Miller, M. I., Sidman, R. L., and Mori, S. (2009). Magnetic resonance imaging and micro-computed tomography combined atlas of developing and adult mouse brains for stereotaxic surgery. Neuroscience 162, 1339-1350. doi: 10.1016/j.neuroscience.2009.05.070

Black, K. J., Snyder, A. Z., Koller, J. M., Gado, M. H., and Perlmutter, J. S. (2001). Template images for nonhuman primate neuroimaging: 1. Baboon. Neuroimage 14, 736-743. doi: 10.1006/nimg.2001.0752

Buffenstein, R. (2008). Negligible senescence in the longest living rodent, the naked mole-rat: insights from a successfully aging species. J. Comp. Physiol. B 178, 439-445. doi: 10.1007/s00360-007-0237-5

Brandt, R., Rohlfing, T., Rybak, J., Krofczik, S., Maye, A., Westerhoff, M., et al. (2005). Three-dimensional average-shape atlas of the honeybee brain and its applications. J. Comp. Neurol. 492, 1-19. doi: 10.1002/cne.20644

Brecht, M., and Freiwald, W. A. (2012). The many facets of facial interactions in mammals. Curr. Opin. Neurobiol. 22, 259-266. doi: 10.1016/j.conb.2011.12.003

Brodmann, K. (2006). Brodmann's Localisation in the Cerebral Cortex, 2006 Edn. New York, NY: Springer.

Buffenstein, R. (2005). The naked mole-rat: a new long-living model for human aging research. J. Gerontol. A, Biol. Sci. Med. Sci. 60, 1369-1377.

Buffenstein, R., and Jarvis, J. U. M. (2002). The naked mole rat: a new record for the oldest living rodent. Sci. Aging Knowledge Environ. 2002, pe7. doi: 10.1126/sageke.2002.21.pe7

Catania, K. C., and Remple, M. S. (2002). Somatosensory cortex dominated by the representation of teeth in the naked mole-rat brain. Proc. Natl. Acad. Sci. U.S.A. 99, 5692-5697. doi: 10.1073/pnas.072097999

Chan, E., Kovacevic, N., Ho, S. K., Henkelman, R. M., and Henderson, J. T. (2007). Development of a high resolution three-dimensional surgical atlas of the murine head for strains $129 \mathrm{~S} 1 / \mathrm{SvImJ}$ and C57Bl/6J using magnetic resonance imaging and micro-computed tomography. Neuroscience 144, 604-615. doi: 10.1016/j.neuroscience.2006.08.080

Chuang, N., Mori, S., Yamamoto, A., Jiang, H., Ye, X., Xu, X., et al. (2011). An MRI-based atlas and database of the developing mouse brain. Neuroimage 54, 80-89. doi: 10.1016/j.neuroimage.2010.07.043

Clarke, F. M., and Faulkes, C. G. (1997). Dominance and queen succession in captive colonies of the eusocial naked mole-rat, Heterocephalus glaber. Proc. R. Soc. B 264, 993-1000. doi: 10.1098/rspb.1997.0137

Conturo, T. E., Lori, N. F., Cull, T. S., Akbudak, E., Snyder, A. Z., Shimony, J. S., et al. (1999). Tracking neuronal fiber pathways in the living human brain. Proc. Natl. Acad. Sci. U.S.A. 96, 10422-10427.

Cooper, H. M., Herbin, M., and Nevo, E. (1993). Visual system of a naturally microphthalmic mammal: the blind mole rat, Spalax ehrenbergi. J. Comp. Neurol. 328, 313-350. doi: 10.1002/cne.903280302

Crish, S. D., Dengler-Crish, C. M., and Catania, K. C. (2006a). Central visual system of the naked mole-rat (Heterocephalus glaber). Anat. Rec. 288, 205-212. doi: 10.1002/ar.a.20288

Crish, S. D., Dengler-Crish, C. M., and Comer, C. M. (2006b). Population coding strategies and involvement of the superior colliculus in the tactile orienting behavior of naked mole-rats. Neuroscience 139, 1461-1466. doi: 10.1016/j.neuroscience.2005.11.073 
Crish, S. D., Rice, F. L., Park, T. J., and Comer, C. M. (2003). Somatosensory organization and behavior in naked mole-rats I: vibrissa-like body hairs comprise a sensory array that mediates orientation to tactile stimuli. Brain Behav. Evol. 62 , 141-151. doi: 10.1159/000072723

Crish, S. D., Dengler-Crish, C. M., and Catania, K. C. (2004). Central visual system of the naked mole-rat. Abstr. Soc. Neurosci. 749, 205-212.

Daw, N. (2006). Visual Development. New York: Springer.

Drager, U. C., and Hubel, D. H. (1975). Physiology of visual cells in mouse superior colliculus and correlation with somatosensory and auditory input. Nature 253, 203-204.

Edrey, Y. H., Hanes, M., Pinto, M., Mele, J., and Buffenstein, R. (2011). Successfu aging and sustained good health in the naked mole rat: a long-lived mammalian model for biogerontology and biomedical research. ILAR J. 52, 41-53.

Franklin, K. B. J., and Paxinos, G. (2007). The Mouse Brain in Stereotaxic Coordinates, 3rd Edn. San Diego, CA: Academic Press.

Fujiyoshi, K., Yamada, M., Nakamura, M., Yamane, J., Katoh, H., Kitamura, K., et al. (2007). In vivo tracing of neural tracts in the intact and injured spinal cord of marmosets by diffusion tensor tractography. J. Neurosci. 27, 11991-11998. doi: 10.1523/jneurosci.3354-07.2007

Giorgio, A., Santelli, L., Tomassini, V., Bosnell, R., Smith, S., De Stefano, N. et al. (2010a). Age-related changes in grey and white matter structure throughout adulthood. Neuroimage 51, 943-951. doi: 10.1016/j.neuroimage.2010. 03.004

Giorgio, A., Watkins, K. E., Chadwick, M., James, S., Winmill, L., Douaud G., etal. (2010b). Longitudinal changes in grey and white matter during adolescence. Neuroimage 49, 94-103. doi: 10.1016/j.neuroimage.2009. 08.003

Good, C. D., Johnsrude, I., Ashburner, J., Henson, R. N., Friston, K. J., and Frackowiak, R. S. (2001). Cerebral asymmetry and the effects of sex and handedness on brain structure: a voxel-based morphometric analysis of 465 normal adult human brains. Neuroimage 14, 685-700. doi: 10.1006/nimg. 2001.0857

Groeschel, S., Vollmer, B., King, M. D., and Connelly, A. (2010). Developmental changes in cerebral grey and white matter volume from infancy to adulthood. Int. J. Dev. Neurosci. 28, 481-489. doi: 10.1016/j.ijdevneu.2010. 06.004

Hattox, A. M., Priest, C. A., and Keller, A. (2002). Functional circuitry involved in the regulation of whisker movements. J. Comp. Neurol. 442, 266-276. doi: 10.1002/cne.10089

Hawrylycz, M., Baldock, R. A., Burger, A., Hashikawa, T., Johnson, G. A., Martone M., et al. (2011). Digital atlasing and standardization in the mouse brain. PLoS Comput. Biol. 7:e1001065. doi: 10.1371/journal.pcbi.1001065

Herold, N., Spray, S., Horn, T., and Henriksen, J. (1998). Measurements of behavior in the naked mole-rat after intraperitonal implantation of a radio-telemetry system. J. Neurosci. Methods 81, 151-158.

Hetling, J. R., Baig-Silva, M. S., Comer, C. M., Pardue, M. T., Samaan, D. Y., Qtaishat, N. M., et al. (2005). Features of visual function in the naked mole-rat Heterocephalus glaber. J. Comp. Physiol. 191, 317-330. doi: 10.1007/s00359-0040584-586

Hikishima, K., Quallo, M. M., Komaki, Y., Yamada, M., Kawai, K., Momoshima S., et al. (2011). Population-averaged standard template brain atlas for the common marmoset (Callithrix jacchus). Neuroimage 54, 2741-2749. doi: 10.1016/j.neuroimage.2010.10.061

Hikishima, K., Sawada, K., Murayama, A. Y., Komaki, Y., Kawai, K., Sato, N. etal. (2013). Atlas of the developing brain of the marmoset monkey constructed using magnetic resonance histology. Neuroscience 230, 102-113. doi: 10.1016/j.neuroscience.2012.09.053

Hjornevik, T., Leergaard, T. B., Darine, D., Moldestad, O., Dale, A. M., Willoch, F., etal. (2007). Three-dimensional atlas system for mouse and rat brain imaging data. Front. Neuroinform. 1:4. doi: 10.3389/neuro.11 .004 .2007

Holmes, M. M., Rosen, G. J., Jordan, C. L., De Vries, G. J., Goldman, B. D., and Forger, N. G. (2007). Social control of brain morphology in a eusocial mammal. Proc. Natl. Acad. Sci. USA 104, 10548-10552. doi: 10.1073/pnas.0610344104

Huang, H., Xue, R., Zhang, J., Ren, T., Richards, L. J., Yarowsky, P., et al. (2009). Anatomical characterization of human fetal brain development with diffusion tensor magnetic resonance imaging. J. Neurosci. 29, 4263-4273. doi 10.1523/jneurosci.2769-08.2009
Huang, H., Zhang, J., Van Zijl, P. C., and Mori, S. (2004). Analysis of noise effects on DTI-based tractography using the brute-force and multi-ROI approach. Magn. Reson. Med. 52, 559-565. doi: 10.1002/mrm.20147

Izraeli, R., and Porter, L. L. (1995). Vibrissal motor cortex in the rat: connections with the barrel field. Exp. Brain Res. 104, 41-54.

Jarvis, J. (1978). Energetics of survival in Heterocephalus glaber (Ruppell), the naked mole rat (Rodentia: Bathyegidae). Bull. Carnegie Mus. Nat. Hist. 6, 81-87.

Jarvis, J. U. (1981). Eusociality in a mammal: cooperative breeding in naked mole-rat colonies. Science 212, 571-573.

Larson, J., and Park, T. J. (2009). Extreme hypoxia tolerance of naked mole-rat brain. Neuroreport 20, 1634-1637. doi: 10.1097/wnr.0b013e32833370cf

Lein, E. S., Hawrylycz, M. J., Ao, N., Ayres, M., Bensinger, A., Bernard, A., et al. (2007). Genome-wide atlas of gene expression in the adult mouse brain. Nature 445, 168-176. doi: 10.1038/nature 05453

May, P. J. (2006). The mammalian superior colliculus: laminar structure and connections. Prog. Brain Res. 151, 321N378. doi: 10.1016/S0079-6123(05)51011-51012

Miyashita, E., and Mori, S. (1995). The superior colliculus relays signals descending from the vibrissal motor cortex to the facial nerve nucleus in the rat. Neurosci. Lett. 195, 69N71.

Mori, S., Crain, B. J., Chacko, V. P., and Van Zijl, P. C. M. (1999). Three-dimensional tracking of axonal projections in the brain by magnetic resonance imaging Ann. Neurol. 45, 265-269. doi: 10.1002/1531-8249(199902)45:2<265::AIDANA21 > 3.0.CO;2-3

Mori, S., Itoh, R., Zhang, J., Kaufmann, W. E., Van Zijl, P. C., Solaiyappan, M., et al. (2001). Diffusion tensor imaging of the developing mouse brain. Magn. Reson. Med. 46, 18N23. doi: 10.1002/mrm.1155

Nikitina, N. V., Maughan-Brown, B., O'Riain, M. J., and Kidson, S. H. (2004) Postnatal development of the eye in the naked mole rat (Heterocephalus glaber). Anat. Rec. 277, 317-337. doi: 10.1002/ar.a.20025

Park, T. J., Comer, C., Carol, A., Lu, Y., Hong, H-S., and Rice, F. L. (2003). Somatosensory organization and behavior in naked mole-rats: II. Peripheral structures, innervation, and selective lack of neuropeptides associated with thermoregulation and pain. J. Comp. Neurol. 465, 104-120. doi: 10.1002/cne. 10824

Park, T. J., Lu, Y., Jüttner, R., Smith, E. S. J., Hu, J., Brand, A., et al. (2008). Selective inflammatory pain insensitivity in the African naked mole-rat (Heterocephalus glaber). PLoS Biol. 6:e13. doi: 10.1371/journal.pbio.0060013

Petiet, A. E., Kaufman, M. H., Goddeeris, M. M., Brandenburg, J., Elmore, S. A., and Johnson, G. A. (2008). High-resolution magnetic resonance histology of the embryonic and neonatal mouse: a 4D atlas and morphologic database. Proc. Natl. Acad. Sci. U.S.A. 105, 12331-12336. doi: 10.1073/pnas.0805 747105

Pietrasanta, M., Restani, L., and Caleo, M. (2012). The corpus callosum and the visual cortex: plasticity is a game for two. Neural Plast. 2012, 838672. doi: $10.1155 / 2012 / 838672$

Pinto, M., Jepsen, K., Trranova, C. J., and Buffenstein, R. (2010). Lack of sexual dimophism in femora of the eusocial and hypogonadic naked mole-rats: a novel animal model for the study of delayed puberty on the skeletal system. Bone 46, 112-120.

Reeve, H. K. (1992). Queen activation of lazy workers in colonies of the eusocial naked mole-rat. Nature 358, 147-149. doi: 10.1038/358147a0

Riccio, A. P., and Goldman, B. D. (2000a). Circadian rhythms of body temperature and metabolic rate in naked mole-rats. Physiol. Behav. 71, 15-22. doi: S00319384(00)00280-8.

Riccio, A. P., and Goldman, B. D. (2000b). Circadian rhythms of locomotor activity in naked mole-rats (Heterocephalus glaber). Physiol. Behav. 71, 1-13. doi: S00319384(00)00281-X.

Sherman, P. W., Jarvis, J. U. M., and Alexander, R. D. (1991). The Biology of the Naked Mole-Rat. Princeton, NJ: Princeton University Press.

Ullmann, J. F., Cowin, G., Kurniawan, N. D., and Collin, S. P. (2010). A threedimensional digital atlas of the zebrafish brain. Neuroimage 51, 76-82. doi: 10.1016/j.neuroimage.2010.01.086

Watanabe, H., Andersen, F., Simonsen, C. Z., Evans, S. M., Gjedde, A., and Cumming, P. (2001). MR-based statistical atlas of the Gottingen minipig brain. Neuroimage 14, 1089-1096. doi: 10.1006/nimg.2001.0910

Wedeen, V. J., Rosene, D. L., Wang, R., Dai, G., Mortazavi, F., Hagmann, P., et al. (2012). The geometric structure of the brain fiber pathways. Science 335, 16281634. doi: $10.1126 /$ science. 1215280 
Xiao, J. (2007). A new coordinate system for rodent brain and variability in the brain weights and dimensions of different ages in the naked mole-rat. J. Neurosci. Methods 162, 162-170. doi: 10.1016/j.jneumeth.2007. 01.007

Xiao, J., Levitt, J. B., and Buffenstein, R. (2006). A stereotaxic atlas of the brain of the naked mole-rat (Heterocephalus glaber). Neuroscience 141, 1415-1435. doi: 10.1016/j.neuroscience.2006.03.077

Zhang, J., Richards, L. J., Yarowsky, P., Huang, H., Van Zijl, P. C., and Mori, S. (2003). Three-dimensional anatomical characterization of the developing mouse brain by diffusion tensor microimaging. Neuroimage 20, 1639-1648.

Conflict of Interest Statement: The authors declare that the research was conducted in the absence of any commercial or financial relationships that could be construed as a potential conflict of interest.
Received: 15 October 2013; accepted: 27 November 2013; published online: 20 December 2013.

Citation: Seki F, Hikishima K, Nambu S, Okanoya K, Okano HJ, Sasaki E, Miura $K$ and Okano $H$ (2013) Multidimensional MRI-CT atlas of the naked mole-rat brain (Heterocephalus glaber). Front. Neuroanat. 7:45. doi: 10.3389/fnana.2013. 00045

This article was submitted to the journal Frontiers in Neuroanatomy.

Copyright (c) 2013 Seki, Hikishima, Nambu, Okanoya, Okano, Sasaki, Miura and Okano. This is an open-access article distributed under the terms of the Creative Commons Attribution License (CC BY). The use, distribution or reproduction in other forums is permitted, provided the original author(s) or licensor are credited and that the original publication in this journal is cited, in accordance with accepted academic practice. No use, distribution or reproduction is permitted which does not comply with these terms. 